\title{
The use of Bayesian inversion to resolve plasma equilibrium ${ }^{\text {a) }}$
}

\author{
M. J. Hole, ${ }^{1, b)}$ G. von Nessi, ${ }^{1}$ D. Pretty, ${ }^{1}$ J. Howard, ${ }^{1}$ B. Blackwell, ${ }^{1}$ J. Svensson, ${ }^{2}$ \\ and L. C. Appel ${ }^{3}$ \\ ${ }^{1}$ Research School of Physics and Engineering, Australian National University, ACT 0200, Australia \\ ${ }^{2}$ Max Planck Institute for Plasma Physics, Teilinstitut Greifswald D17491, Germany \\ ${ }^{3}$ Euratom/CCFE, Culham Science Centre, Abingdon, Oxon OX143DB, United Kingdom
}

(Presented 19 May 2010; received 18 May 2010; accepted 2 August 2010; published online 27 October 2010)

\begin{abstract}
Recently, Bayesian probability theory has been used at a number of experiments to fold uncertainties and interdependencies in the diagnostic data and forward models, together with prior knowledge of the state of the plasma, to increase accuracy of inferred physics variables. A new probabilistic framework, MINERVA, based on Bayesian graphical models, has been used at JET and W7-AS to yield predictions of internal magnetic structure. A feature of the framework is the Bayesian inversion for poloidal magnetic flux without the need for an explicit equilibrium assumption. Building on this, we discuss results from a new project to develop Bayesian inversion tools that aim to (1) distinguish between competing equilibrium theories, which capture different physics, using the MAST spherical tokamak, and (2) test the predictions of MHD theory, particularly mode structure, using the H-1 Heliac. Specifically, we report on correction of the motional Stark effect, pickup coils, flux-loop constrained Bayesian inferred equilibrium for varying toroidal flux. (C) 2010 American Institute of Physics. [doi:10.1063/1.3491044]
\end{abstract}

\section{INTRODUCTION}

Due to the increase in plasma external heating, high performance magnetically confined fusion plasmas have drifted far from the simple picture of ideal magnetohydrodynamics (MHD), which describes the plasma as a single, stationary, isotropic Maxwellian fluid. Several important observed deviations include temperature anisotropy, substantial rotation, and significant stored energy residing in the energetic particle population produced by charge exchange of fast beams with thermals. ${ }^{1}$

A parallel development has been the improvement in the diversity, accuracy, and resolution of plasma diagnostics. Interpretation, however, often requires a detailed knowledge of the plasma equilibrium. For example, inference of the toroidal current profile $j_{\phi}(\psi)$ from line of sight measurements of the polarization angle requires knowledge of the poloidal flux $\psi$ across the plasma. A widespread technique used in this current tomography problem is least-squares fitting of the plasma parameters $\mathcal{I}$ to the measurement vector $\mathcal{D}$, in which prior assumptions, such as the position of the poloidal flux surfaces, are included via a penalty term in the fit.

The confluence of higher performance plasmas with diagnostic improvements has led to an anomaly: data and kinetic simulations are sometimes inconsistent with static ideal MHD equilibria. For instance, magnetic reconstruction based on static ideal MHD ignores flux surface deviations of electron density in Thomson scattering (TS) data, which

\footnotetext{
a) Contributed paper, published as part of the Proceedings of the 18th Topical Conference on High-Temperature Plasma Diagnostics, Wildwood, New Jersey, May 2010.

b) Electronic mail: matthew.hole@ anu.edu.au.
}

arise due to toroidal rotation, as well as the energetic complexity of the plasma, which has a non-Gaussian distribution function.

Recently, a new integrated data-modeling approach for inference of fusion plasma parameters has emerged which offers a natural framework with which to resolve different physics models. The Bayesian approach to inference in fusion plasmas, developed by multiple authors, ${ }^{2-9}$ involves the specification of an initial prior probability distribution function (pdf), $P(\mathcal{I})$, which is then updated by taking into account information that the measurements provide through the likelihood pdf $P(\mathcal{D} \mid \mathcal{I})$. The result is the posterior distribution $P(\mathcal{I} \mid \mathcal{D})$ given by Bayes' formula

$$
P(\mathcal{I} \mid \mathcal{D})=P(\mathcal{D} \mid \mathcal{I}) P(\mathcal{I}) / P(\mathcal{D}) .
$$

The advantage of the Bayesian approach over traditional inversion techniques is twofold: (i) prior knowledge, including known parameter interdependencies is made explicit, and (ii) as the formulation is probabilistic, random errors, systematic uncertainties, and instrumental bias are integral part of the analysis rather than an afterthought.

We have implemented Bayesian inversion using the MINERVA framework. ${ }^{10}$ In this framework, probabilistic graphical models are used to project the dependence of the posterior distribution function on the prior, the data, and the likelihood. An advantage of this approach is that it visualizes the complex interdependency between data and model, and thus expedites model development. In this paper we update earlier results of Bayesian inference of force balance in the mega-ampere spherical tokamak. ${ }^{11}$ 


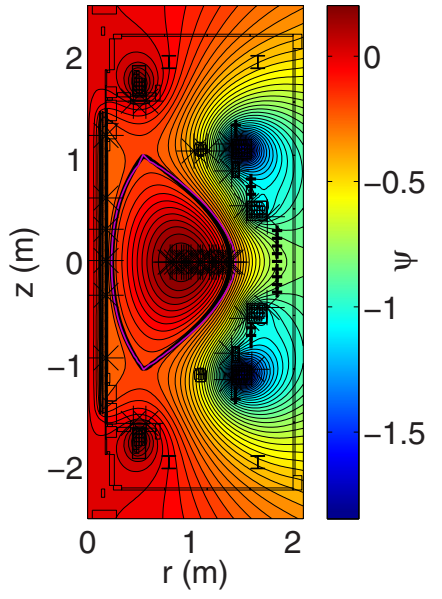

FIG. 1. (Color online) Poloidal flux surfaces inferred for MAST shot no. 22254 at $320 \mathrm{~ms}$ using pickup coils, flux loops, and MSE. The last closed flux surface from the plasma beam model/EFIT is plotted in heavy black/ purple.

\section{MAST AND CURRENT TOMOGRAPHY}

The mega-ampere spherical tokamak is one of the world's largest spherical tokamaks. Although Mega-Ampere Spherical Tokamak (MAST) physics and technology development have contributed across a broad range of fusion science, two properties of MAST help motivate this research: high performance and precision diagnostics.

Recently, both MAST neutral beam injectors have been upgraded to 3.8 MW. This has enabled plasma performance to be routinely lifted above $\beta_{n} \approx 5$, which was reported in $2005 .{ }^{1}$ MAST is also equipped with an array of precision diagnostics, ${ }^{12}$ including a high spatial resolution, single-time point, ruby TS system, a multitime point Nd:YAG TS system with a coarser spatial resolution, motional Stark effect (MSE), charge exchange recombination, and fast magnetics. $^{13}$

Our development of Bayesian inference of the current profile on MAST, detailed in Hole et al. ${ }^{11}$ closely follows the seminal work of Svensson and Werner. ${ }^{7}$ In that work, the plasma was represented as a grid of toroidal axis-symmetric current beams, each with rectangular cross-section and each beam carrying a uniform current density. In MAST, we have placed these beams so as to fill-out the entire plasma volume, including regions outside the last closed flux surface up to the vacuum poloidal field coils. The magnetic field generated is then a summation of Biot-Savart's law over current beams.

Figure 1 shows poloidal flux surfaces from MAST discharge no. 22254 at $320 \mathrm{~ms}$ using pickup coils, flux loops and MSE data. Discharge no. 22254 is a deuterium plasma in a double-null configuration, which was heated with $3.1 \mathrm{MW}$ of neutral beam heating and a plasma current of $I_{p}$ $=800 \mathrm{kA}$. The time of $320 \mathrm{~ms}$ analyzed here is the highresolution TS time closest to the peak $\beta$ for this shot. The figure shows a contour plot of $\psi(R, Z)$ which is calculated from the maximum of the posterior of the distribution of toroidal current beams. Overlaid on the contours are traces of the poloidal field coil cross sections and conducting surface cross sections for the MAST experiment, as well as the last closed flux surface calculated from the plasma beam model

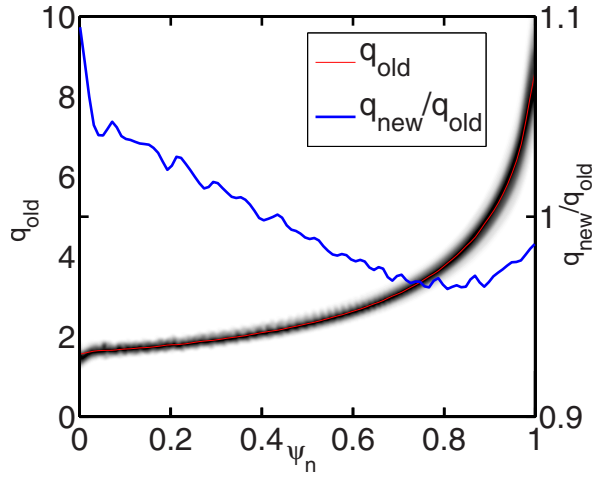

FIG. 2. (Color online) Safety factor, $q$, profile as a function of normalized poloidal flux found by sampling the posterior 200 times for shot no. 22254 at $320 \mathrm{~ms}$. The poloidal flux is normalized such that $\psi_{n}=0$ is the magnetic axis and $\psi_{n}=1$ is the edge. Also shown is $q_{\text {old }} / q_{\text {new }}$, obtained by correcting the toroidal flux function to account for poloidal currents.

and the corresponding EFIT last closed flux surfaces. One outcome of the Bayesian approach is generation of pdfs of inferred quantities from which the uncertainty can be inferred. For instance, Fig. 2 shows the corresponding safety factor or $q$ profile and its uncertainty. In Bayesian inference, the uncertainty in an inferred parameter is computed by sampling different realizations from the (generally not analytically tractable) posterior. Here, we have computed the $q$ profile for each realization of current beams across the plasma and rendered the ensemble of samples as a histogram, where the shading represents the density of samples.

While not shown here, we have also computed the poloidal flux surfaces for two other MAST discharges: nos. 25087 and 24600 at $320 \mathrm{~ms}$, which was near the peak $\beta$ for these shots. Both discharges had similar neutral beam heating and plasma current to no. 22254. We have selected these high performance discharges to maximize plasma poloidal currents, and hence maximize the correction to the toroidal flux from the vacuum field, which is investigated in Sec. III.

\section{INFERENCE OF TOROIDAL FLUX}

A physics goal of our work is to exploit the improved resolution of diagnostics to infer the validity of different force balance descriptions. Specifically, we have in mind development of a framework that will validate energetic plasma resolved force balance models. To build toward this goal, we apply Bayesian inference to the axis-symmetric GradShafranov equilibrium description of ideal MHD force balance, $\mathbf{J} \times \mathbf{B}=\nabla P$.

The Grad-Shafranov equation ${ }^{14}$ can be written $F(R, Z)$ $=0$, where

$$
\begin{aligned}
& F(R, Z)=-\mu_{0} R j_{\phi}(\psi)+\mu_{0} R^{2} p^{\prime}(\psi)+\mu_{0}^{2} f(\psi) f^{\prime}(\psi), \\
& -\mu_{0} R j_{\phi}(\psi)=R \frac{\partial}{\partial R} \frac{1}{R} \frac{\partial \psi}{\partial R}+\frac{\partial^{2} \psi}{\partial z^{2}} .
\end{aligned}
$$

For a real plasma, the presence of nonideal effects will mean $F(R, Z)$ is nonzero. Our long term aim is to compute $P(\mathcal{F} \mid \mathcal{D})$ using Bayes formula, such that $P(\mathcal{F} \mid \mathcal{D})$ $=P(\mathcal{D} \mid \mathcal{F}) P(\mathcal{F}) / P(\mathcal{D})$. Here, the vector $\mathcal{D}$ comprises all the magnetics, MSE, TS, and charge exchange recombination data, while the column vector $\mathcal{F}$ contains $F(R, Z)$ evaluated 
from Eq. (2) at different $(R, Z)$ across the plasma. To date, we have implemented MSE and magnetic diagnostics in MINERVA and work is in progress to capture TS and charge exchange recombination spectroscopy.

In the absence of a full model some progress can however be made if we assume the plasma obeys ideal force balance and $p^{\prime}(\psi)$ is assumed to be independent of $f(\psi) f^{\prime}(\psi)$ and $j_{\phi}(\psi)$. In this instance, and providing we are able to estimate $p^{\prime}(\psi)$ and its distribution from measurements, then $f(\psi) f^{\prime}(\psi)$ across the midplane can be computed through Eq. (2). As a first step to inference of force balance in a real plasma, we compute $f(\psi) f^{\prime}(\psi)$, integrate to find $f(\psi)$, and substitute this back into MINERVA. By examining the changes in the position of the magnetic axis and $q$ profile from the recomputed solution, we are able to quantify the impact of poloidal currents on current tomography without the need for a separate magnetic reconstruction by EFIT. This folds $p^{\prime}(\psi)$ into $j_{\phi}(\psi)$ through the influence of poloidal currents.

In Hole et al. ${ }^{11}$ we described our approach to compute $p^{\prime}(\psi)$ and its distribution from TS data. The process involved the following steps. (1) Assume that the mean values of density and temperature satisfy $n_{i} / n_{e}=0.8$ and $T_{i} / T_{e}=1.1$, as typically extracted from a charge exchange recombination measurement. We have also assumed the same distribution of data as TS. (2) Generate $T_{e}, T_{i}, n_{e}, n_{i}$ samples that satisfy the prescribed pdfs $P\left(n_{e}\right), P\left(T_{e}\right), P\left(n_{i}\right), P\left(T_{i}\right)$. (3) Map each sample $p(r)$ to $p(\psi)$ using $\psi(r)$ across the inboard chord determined from MSE. (4) Fit a fourth order polynomial in normalized flux to find a smoothed $p(\psi)$. (5) Repeat steps (2)-(4) until the pdf for $p(\psi)$ no longer changes.

Using the pressure profile fit, we have sampled $p^{\prime}(\psi)$ and $j_{\phi}$ across the midplane and computed $f(\psi) f^{\prime}(\psi)$, and then integrated to obtain $f(\psi)$. This yields a toroidal flux profile that varies approximately linearly in poloidal flux: for no. $22254 f(\psi)$ varies from -0.407 , its vacuum value at the edge, to -0.496 at the core. That is, poloidal plasma currents are paramagnetic, and increase $B_{\phi}$ and hence $q_{0}$ at the core. Figure 2 shows the change in the safety factor profile, $q_{\text {new }} / q_{\text {old }}$, computed from MINERVA if the toroidal flux is updated with that inferred from force balance. While $q_{0}$ increases near the core, the increase is less than the increase in $f(\psi)$ : this is because for fixed polarization angle, an increase in $B_{\phi}$ requires an increase in $B_{z}$, and hence $B_{\theta}$. Thus, all closed poloidal flux surfaces shift outward. In the core region, where the magnetic shear is small, this poloidal flux expansion has little effect on $q$. In the edge region however, which has large magnetic shear, the effect of poloidal flux expansion is the dominant effect on $q$, causing it to reduce. Finally, at the plasma-boundary, the toroidal flux approaches the vacuum, and so $q_{\text {new }} / q_{\text {old }}$ approaches unity. The oscillations in $q_{\text {new }} / q_{\text {old }}$, as well as the behavior at the core, is a result of finite number of toroidal current beams and finite number of inferred poloidal flux surfaces. This fine structure decreases with increasing grid resolution. Similar results for discharge nos. 25087 and 24600 are obtained. This is the first time poloidal current correction has been included in the MINERVA Bayesian inference inversion on MAST.

\section{CONCLUSIONS}

In this work we have implemented a Bayesian inversion framework for inference of poloidal flux surfaces and used it, together with the constraint that the plasma obeys ideal MHD force balance, to infer the correction to the toroidal flux function due to poloidal currents for three high performance MAST discharges. When substituted back into MINERVA, we find that the on-axis safety factor increases by $\approx 5 \%$ and the safety factor at the $\psi_{n}=0.8$ surfaces decreases by $\approx 5 \%$. This is the first time poloidal current correction has been included in the MINERVA Bayesian inference inversion on MAST. In ongoing work we are developing forward models of Thomson scattering and charge exchange recombination spectroscopy for inference of force balance in MAST plasmas and developing Bayesian inference models for mode structure in $\mathrm{H}-1$ plasmas.

\section{ACKNOWLEDGMENTS}

This work was jointly funded by the Australian Government through International Science Linkages Grant No. CG130047, the Australian National University, the United Kingdom Engineering and Physical Sciences Research Council, and by the European Communities under the contract of Association between EURATOM and CCFE.

${ }^{1}$ M. J. Hole, R. J. Akers, L. C. Appel, R. J. Buttery, C. Brickley, N. J. Conway, M. Gryaznevich, T. C. Hender, O. J. Kwon, M. Valovic, S. Medvedev, A. Patel, S. Saarelma, D. Taylor, H. R. Wilson, and MAST Team, Plasma Phys. Controlled Fusion 47, 581 (2005).

${ }^{2}$ G. A. Cottrell, Maximum Entropy in Action (Oxford Science, Oxford, 1990).

${ }^{3}$ A. P. Millar, D. C. McDonald, and D. A. Diver, Plasma Phys. Controlled Fusion 42, 337 (2000)

${ }^{4}$ R. Fischer, A. Dinklage, and E. Pasch, Plasma Phys. Controlled Fusion 45, 1095 (2003).

${ }^{5}$ A. Dinklage, R. Fischer, and J. Svensson, Fusion Sci. Technol. 46, 355 (2004).

${ }^{6}$ J. Svensson, A. Dinklage, J. Geiger, A. Werner, and R. Fischer, Rev. Sci. Instrum. 75, 4219 (2004).

${ }^{7}$ J. Svensson and A. Werner, Plasma Phys. Controlled Fusion 50, 085002 (2008)

${ }^{8}$ O. Ford, J. Svensson, A. Boboc, D. C. McDonald, and JET-EFDA Contributors, Rev. Sci. Instrum. 79, 10F324 (2008).

${ }^{9}$ M. Reginatto and A. Zimbal, Rev. Sci. Instrum. 79, 023505 (2008).

${ }^{10}$ J. Svensson and A. Werner, Proceedings of the IEEE International Symposium on Intelligent Signal Processing WISP, October 2007.

${ }^{11}$ M. J. Hole, G. von Nessi, J. Bertram, J. Svensson, L. C. Appel, B. D. Blackwell, R. L. Dewar, and J. Howard, J. Plasma Fusion Res. 9, 479 (2010).

${ }^{12}$ H. Meyer, R. J. Akers, F. Alladio et al., Nucl. Fusion 49, 104017 (2009).

${ }^{13}$ M. J. Hole, L. C. Appel, and R. Martin, Rev. Sci. Instrum. 80, 123507 (2009).

${ }^{14}$ J. Wesson, Tokamaks, 2nd ed. (Oxford University Press, Oxford, 1997). 